\title{
Produção de biocompostos com atividade antimicrobiana de Streptomyces sp. ante isolados de mastite caprina
}

\author{
Production of biocompounds with antimicrobial activity of Streptomyces $s p$. \\ Isolated in face of goat mastitis] \\ T. Pajeú Nascimento ${ }^{1}$, C.S. Porto ${ }^{1}$, M.F.S. Teixeira ${ }^{2}$, T.S. Porto ${ }^{3}$, A.L.F. Porto ${ }^{1 *}$ \\ ${ }^{1}$ Universidade Federal Rural de Pernambuco - Recife, PE \\ ${ }^{2}$ Universidade Federal do Amazonas - Coroado Manaus, AM \\ ${ }^{3}$ Unidade Acadêmica de Garanhuns - Universidade Federal Rural de Pernambuco - Garanhuns, PE
}

\begin{abstract}
RESUMO
Actinomicetos são um dos principais produtores de enzimas, vitaminas e metabólitos secundários, destacando-se o gênero Streptomyces, o qual tem uma ampla capacidade de produção de antibióticos eficazes no combate a diferentes microrganismos, entre eles o Staphylococcus sp. Em virtude dessa eficiência no combate a patógenos, o objetivo deste trabalho foi avaliar a produção de metabólitos com atividade antimicrobiana produzidos por 67 Streptomyces isolados de liquens da região amazônica, ante isolados de mastite caprina (Staphylococcus aureus) do estado de Pernambuco, Brasil. Foi utilizado um planejamento fatorial para avaliar a influência das fontes de carbono (glicose) $0 \%, 0,5 \%$ e $1 \%$ e de nitrogênio (farinha de soja) $1 \%, 2,5 \%$ e $4 \%$ na produção dos antimicrobianos, bem como das variáveis $\mathrm{pH}$, biomassa e atividade antimicrobiana. Dos Streptomyces estudados, o DPUA 1566 foi o que se destacou por formação de halos de inibição entre 18 e $26 \mathrm{~mm}$ ante os isolados de mastite caprina. Foi possível verificar que a fonte de carbono inibiu a produção de antimicrobianos quando submetidos a uma concentração de glicose de 1\%; com a retirada desta, os Streptomyces apresentaram uma elevada capacidade de produção de metabólitos com atividade antimicrobiana tendo potencial para o tratamento de mastite caprina.
\end{abstract}

Palavras-chave: cabra, actinomicetos, Staphylococcus aureus, mastite

\begin{abstract}
Actinomycetes are a leading producer of enzymes, vitamins and secondary metabolites, especially the genus Streptomyces, which have a large capacity for the production of natural antibiotics and are effective against various micro-organisms including Staphylococcus sp. Due to this efficiency in combating micro-organisms, the aim of this study was to evaluate the production of metabolites with antimicrobial activity produced by Streptomyces isolated from lichens 67 in the Amazonia, compared to isolates from goat mastitis (Staphylococcus aureus) in the state of Pernambuco, Brazil. We used a complete factorial design to evaluate the influence of concentrations of carbon sources (glucose) at $0 \%$, $0.5 \%$ and $1 \%$ and nitrogen (soybean flour) at $1 \%, 2.5 \%$ and $4 \%$ in the production of antimicrobial metabolites, and the influence of the $\mathrm{pH}$, microbial biomass and activity variables. Of the studied Streptomyces DPUA 1566 what stood out was the formation of inhibition halos between 18 to $26 \mathrm{~mm}$ compared to the isolates from goats mastitis. It was noted that the carbon source inhibited the production of antimicrobial metabolites when subjected to a glucose concentration of 1\%. However, after the discontinuation, Streptomyces showed a high capacity to produce metabolites with antimicrobial activity, which has an excellent potential for the treatment of mastitis in goats.
\end{abstract}

Keywords: goat, Actinomycetes, Staphylococcus aureus, mastitis

Recebido em 5 de julho de 2012

Aceito em 8 de maio de 2013

*Autor para correspondência (corresponding author)

E-mail: analuporto@yahoo.com.br 


\section{INTRODUÇÃO}

O Streptomyces é o principal gênero utilizado para a produção de antibióticos. O número de compostos antimicrobianos declarados oriundos de espécies desse gênero por ano aumentou quase exponencialmente em cerca de três décadas, demonstrando, assim, que esse grupo de microrganismos permanece sendo uma fonte promissora para a produção de novos agentes antimicrobianos. Devido a sua alta capacidade de produzir uma grande variedade de metabólitos (antibióticos, vitaminas e enzimas), os Streptomyces e outros actinomicetos são responsáveis por cerca de três quartos de todos os produtos conhecidos (Vijayakumar et al., 2011; Gama et al., 2012; Pacheco da Rosa et al., 2013).

O desenvolvimento de fármacos eficientes no combate a infecções bacterianas revolucionou o tratamento médico, ocasionando a redução drástica da mortalidade causada por doenças microbianas (Rang et al., 2003; Silveira et al., 2006). Entretanto, o uso inadequado e excessivo desses antimicrobianos leva ao desenvolvimento de microrganismos resistentes, tornando-se uma ameaça para a saúde pública.

Acredita-se que uma grande parte da virulência do $S$. aureus se deve à atuação das exotoxinas, enterotoxinas e enterotoxinas como proteínas, além de essa infecção induzir a ativação aberrante das células $\mathrm{T}$, suprimindo a resposta imune (Foster, 2005; Park et al., 2006; Mork et al., 2010).

Muitas infecções hospitalares, comunitárias e intoxicações são causadas principalmente por bactérias do gênero Staphylococcus, além de algumas doenças de importância veterinária, como a mastite. É a principal doença contagiosa em diversos rebanhos, como caprinos e bovinos, a qual constitui uma inflamação da glândula mamária, que aumenta a contagem de células somáticas (CSS) e diminui a produção e a qualidade do leite (Peixoto et al., 2013). A mastite é a principal doença dos rebanhos leiteiros no mundo inteiro, causando prejuízos econômicos tanto aos pequenos produtores de leite quanto à indústria de lacticínios, afetando toda a cadeia produtiva (Tozzeti et al., 2008; Mota et al., 2012).
A mastite pode reduzir a quantidade e a qualidade do leite produzido; o seu agravamento pode levar à perda total da capacidade secretora da glândula mamária (Ribeiro et al., 2003), somando-se a isso prejuízos ocasionados com gastos com medicamentos e assistência veterinária, inutilização de leite contaminado após tratamento, descarte precoce de animais doentes, o que pode prejudicar a cultura desses animais (Freitas et al., 2005; Cunha et al., 2006). A mastite clínica caprina é causada por $S$. coagulase positiva ( $S$. aureus), e a mastite subclínica, sobretudo, por $S$. coagulase negativa (SCN), sendo os principais SCN: S. epidermidis, $S$. chromogenes e S. simulans, $S$. caprae e $S$. agalactiae (Contreras et al., 2003 e Neves et al., 2010). A literatura evidencia que o $S$. aureus é o maior causador de mastite em ruminantes incluindo cabras leiteiras, acarretando casos mais severos de mastite (Taponen e Pyorala, 2009; França et al., 2012).

Em face da necessidade de desenvolvimento de agentes antimicrobianos eficientes ante agentes patogênicos resistentes, o presente trabalho tem como objetivo selecionar um Streptomyces isolado de liquens da região amazônica, produtor de biocompostos com atividade antimicrobiana, ante isolados de mastite caprina do estado de Pernambuco.

\section{MATERIAL E MÉTODOS}

As amostras de Streptomyces foram isoladas de liquens da região amazônica, pertencentes à coleção de culturas DPUA (Departamento de Parasitologia da Universidade Federal do Amazonas), totalizando 67 isolados. Os isolados foram mantidos no meio ISP-2 (Pridham et al., 1957), semeados em placas de Petri incubadas a $30^{\circ} \mathrm{C}$ por 168 horas e preservados pelo método de Castellani (Castellani, 1939). Para a esporulação, foi utilizado meio ISP-2 modificado pela retirada de glicose.

Os microrganismos teste foram Staphylococcus aureus isolados de mastite caprina pertencente a rebanhos de fazendas da região agreste do estado de Pernambuco-Brasil. O ágar nutriente (AN) foi utilizado como o meio de manutenção, e os microrganismos foram preservados como cultura estoque em criotubos utilizando-se glicerol $10 \%$ (v/v), mantidos a $-20^{\circ} \mathrm{C}$. 
A produção de metabólitos foi estimada por meio da técnica de bloco de gelose (Brown et al., 1976), utilizando-se Staphylococcus aureus como microrganismo teste. Foi usado o meio de cultura ágar Müeller Hinton em placas de Petri (90x15mm de diâmetro). A superfície foi semeada em uma suspensão das células de $S$. aureus crescido previamente em TSB (Tryptic Soy Broth) por 24 horas, na concentração de $10^{8}$ $\mathrm{UFC} / \mathrm{mL}$.

Em cada placa teste foram sobrepostos fragmentos de $6 \mathrm{~mm}$, retirados da área central das culturas dos actinomicetos crescidos em meio ISP-2 sólido, que foi modificado pela retirada da glicose a $30^{\circ} \mathrm{C}$ por 168 horas, e incubados por 24 horas a $37^{\circ} \mathrm{C}$. A produção de biocompostos com atividade antimicrobiana foi verificada pela formação de halos claros de inibição do crescimento microbiano e expressos em milímetros (mm). O efeito inibitório ante os microrganismos testados foi classificado de acordo com Sahin e Ugur (2003), sendo o tamanho da zona de inibição dividido em quatro grupos distintos: grupo passivo - zona de inibição menor que $10 \mathrm{~mm}$; grupo ligeiramente ativo - zona de inibição entre 11 e $20 \mathrm{~mm}$; grupo moderadamente ativo - zona de inibição entre 21 e $30 \mathrm{~mm}$; e grupo altamente ativo, cuja zona de inibição é maior que $31 \mathrm{~mm}$.

Para a produção de metabólitos com atividade antimicrobiana pelo actinomiceto selecionado, foi utilizado o meio MS-2 (Porto et al., 1996); para a produção dos metabólitos com atividade antimicrobiana, este foi composto por: 2,0\% $(\mathrm{p} / \mathrm{v})$ de farinha de soja, $0,06 \%$ (p/v) de $\mathrm{MgSO}_{4} .7 \mathrm{H}_{2} \mathrm{O}, \quad 0,1 \% \quad(\mathrm{p} / \mathrm{v}) \quad \mathrm{deNH}_{4} \mathrm{Cl}, \quad 0,435 \%$ $(\mathrm{p} / \mathrm{v})$ de $\mathrm{K}_{2} \mathrm{HPO}_{4}$ e $0,1 \mathrm{~mL}$ de solução mineral (100mg de $\mathrm{FeSO}_{4}, \mathrm{MnCl}_{2}, 4 \mathrm{H}_{2} \mathrm{O}, \mathrm{ZnSO}_{4}, \mathrm{CaCl}_{2}$. $\mathrm{H}_{2} \mathrm{O}$ em $100 \mathrm{~mL}$ de água destilada, com $\mathrm{pH}$ inicial 7,0) modificado pela retirada da glicose.
Foram utilizados Erlenmeyers $(250 \mathrm{~mL})$ contendo $60 \mathrm{~mL}$ de meio de cultura, o inóculo de $10^{8} \mathrm{UFC} / \mathrm{mL}$ foi incubado em agitador orbital (200rpm) a $28^{\circ} \mathrm{C}$. O crescimento celular, a curva de $\mathrm{pH}$ e a determinação da atividade antimicrobiana foram determinados após 24,48 , 72, 96 e 120 horas de fermentação.

A atividade antimicrobiana foi estimada empregando-se o método da difusão em disco descrito por Ericsson e Sherris (1971) utilizandose $S$. aureus, isolado de mastite caprina do estado de Pernambuco. Os sobrenadantes aliquotados a cada $24 \mathrm{~h}$ durante a fermentação em MS-2 do isolado de Streptomyces sp. DPUA 1566 previamente selecionado foram utilizados para impregnar os discos de papel-filtro (com 5mm) e aplicados na superfície da placa teste $(24 \mathrm{~h}$ a $\left.37^{\circ} \mathrm{C}\right)$

Os ensaios foram realizados como recomendado pelas normas da NCCLS (National..., 2003), com o meio de cultura ágar Müeller Hinton, onde foi inoculada, com auxílio de um swab estéril, uma suspensão dos microrganismos teste $\left(10^{8} \mathrm{UFC} / \mathrm{mL}\right)$ crescidos em TSB por 24 horas. A atividade antimicrobiana foi detectada pela presença de zonas de inibição expressa em milímetros, considerando-se os valores médios das duas repetições.

Os estudos de produção foram realizados com um auxílio de um planejamento fatorial $2^{2} \mathrm{com}$ quatro pontos centrais, avaliando-se as concentrações de fontes de nitrogênio e de carbono (Tab. 1). As variáveis respostas foram crescimento da biomassa, $\mathrm{pH}$ e atividade antimicrobian. Todos os resultados foram analisados com auxílio do software Statistica 8.0 (Statsoft Inc, 2008).

Tabela 1. Níveis das variáveis estudadas no planejamento fatorial completo $2^{2}$ para a produção de biocompostos por Streptomyces sp. DPUA1566

\begin{tabular}{lccc}
\multicolumn{1}{c}{ Fatores } & \multicolumn{3}{c}{ Níveis } \\
\cline { 2 - 4 } & Inferior $(-1)$ & Central $(0)$ & Superior $(+1)$ \\
\hline $\begin{array}{l}\text { Concentração de } \\
\text { filtrado de soja (\%) }\end{array}$ & 1,0 & 2,5 & 4,0 \\
$\begin{array}{l}\text { Concentração de } \\
\text { glicose }(\%)\end{array}$ & 0 & 0,5 & 1,0 \\
\hline
\end{tabular}




\section{RESULTADOS E DISCUSSÃO}

Foram analisados 67 isolados de Streptomyces para produção de metabólitos com atividade antimicrobiana, dos quais 10 apresentaram produção de biocompostos com atividade antimicrobian ante os $S$. aureus isolados de mastite caprina, correspondendo a aproximadamente $17 \%$ do total de isolados. As atividades foram verificadas com a presença de halos de inibição, os quais variaram de 10 a $26 \mathrm{~mm}$.

O espectro de ação das biomoléculas obtidas a partir de Streptomyces ante os isolados de mastite caprina apresentou perfil de inibição semelhante às atividades antimicrobianas dos Streptomyces, sendo estas classificadas em diversos grupos, de acordo com a Tab. 2.

Tabela 2. Seleção dos Streptomyces produtores de antibióticos

\begin{tabular}{|c|c|c|c|c|c|}
\hline \multirow[b]{2}{*}{ Streptomyces } & \multicolumn{5}{|c|}{ Efeito inibitório do Streptomyces ante os Staphylococcus aureus } \\
\hline & $\begin{array}{c}\text { Altamente } \\
\text { ativo }\end{array}$ & Ativo & $\begin{array}{c}\text { Levemente } \\
\text { ativo }\end{array}$ & Passivo & Sem efeito \\
\hline $\begin{array}{c}\text { DPUA } 1542 \\
\text { Streptomyces sp. }\end{array}$ & & & $75 \%$ & --------- & $25 \%$ \\
\hline $\begin{array}{c}\text { DPUA } 1543 \\
\text { Streptomyces sp. } \\
\text { DPUA } 1549\end{array}$ & & & $50 \%$ & $25 \%$ & $25 \%$ \\
\hline $\begin{array}{c}\text { Streptomyces sp. } \\
\text { DPUA } 1550\end{array}$ & & & $100 \%$ & & \\
\hline $\begin{array}{l}\text { Streptomyces sp. } \\
\text { DPUA } 1560\end{array}$ & & & $75 \%$ & ---------- & $25 \%$ \\
\hline $\begin{array}{l}\text { Streptomyces sp. } \\
\text { DPUA1565 }\end{array}$ & & & $50 \%$ & $25 \%$ & $25 \%$ \\
\hline $\begin{array}{l}\text { Streptomyces sp. } \\
\text { DPUA } 1566\end{array}$ & & $50 \%$ & $50 \%$ & --------- & ---------- \\
\hline $\begin{array}{l}\text { Streptomyces sp. } \\
\text { DPUA } 1568 \\
\text { Streptomyces sp. } \\
\text { DPUA } 1573\end{array}$ & & & $75 \%$ & --------- & $25 \%$ \\
\hline Streptomyces sp. & & & $75 \%$ & $25 \%$ & --------- \\
\hline $\begin{array}{c}\text { DPUA } 1589 \\
\text { Streptomyces sp. }\end{array}$ & & & & $50 \%$ & $50 \%$ \\
\hline
\end{tabular}

Resultados obtidos por Sahin e Ugur (2003) corroboram os resultados relatados neste estudo, em que classificaram o Streptomyces isolates como moderadamente ativo ante os Staphylococcus xylosus (MU29), S. epidermidis (MU30), S. aureus (ATCC6538/P) e S. aureus (MU38), os quais formaram zonas de inibição maiores que $20 \mathrm{~mm}$. Mitra et al. (2011), ao estudarem 11 actinomicetos isolados de solo de estuário da ilha de Sagar, Índia, obtiveram, assim como os resultados apresentados neste trabalho, uma atividade antimicrobiana ante os $S$. aureus (MTCC 96) de 11 a $23 \mathrm{~mm}$ de zona de inibição, confirmando os resultados encontrados na literatura.
Entre os actinomicetos estudados, o que apresentou melhor atividade antimicrobiana após 24 horas foi o Streptomyces sp. DPUA 1566, o qual obteve halos de inibição de $26 \mathrm{~mm}$ ante os isolados de mastite caprina. O tamanho do halo de inibição de 26mm, obtido (DPUA 1566) encontra-se dentro dos padrões já descritos pela literatura. Al-Zahrani (2007) e colaboradores estudaram Streptomyces sp., isolado de Jazan (J12) ante os S. aureus e Bacillus subtilis, e obtiveram halos de inibição de $26 \mathrm{~mm}$ e $25 \mathrm{~mm}$.

Os metabólitos secundários, como, por exemplo, os antibióticos, são sintetizados por várias vias metabólicas e também por espécies geneticamente distintas, sendo sua produção afetada por diferentes condições ambientais. 
Parâmetros da fermentação, tais como: tempo, temperatura, $\mathrm{pH}$ e nutrientes, podem ser modificados visando ampliar a quantidade dos metabólitos secundários produzidos (Pfefferle et al., 2000). Em virtude desses parâmetros, foram estudados os componentes do meio de cultivo, ou seja, foram modificadas as concentrações da fonte de carbono (glicose) e da fonte de nitrogênio (farinha de soja) na produção de antibiótico por Streptomyces sp. DPUA 1566, sendo utilizado um planejamento fatorial completo para avaliação da influência desses componentes na produção do metabólito antimicrobiano (Tab. 1).

Foram observadas a fase característica de crescimento microbiano do Streptomyces DPUA 1566 em meio MS-2, a fase lag de 24 horas, ou seja, o período de adaptação do microrganismo ao meio de cultura, a fase exponencial característica com crescimento microbiano máximo em 72 horas e, em seguida, a fase estacionária e a de declínio com 120 horas de fermentação (Fig. 1).

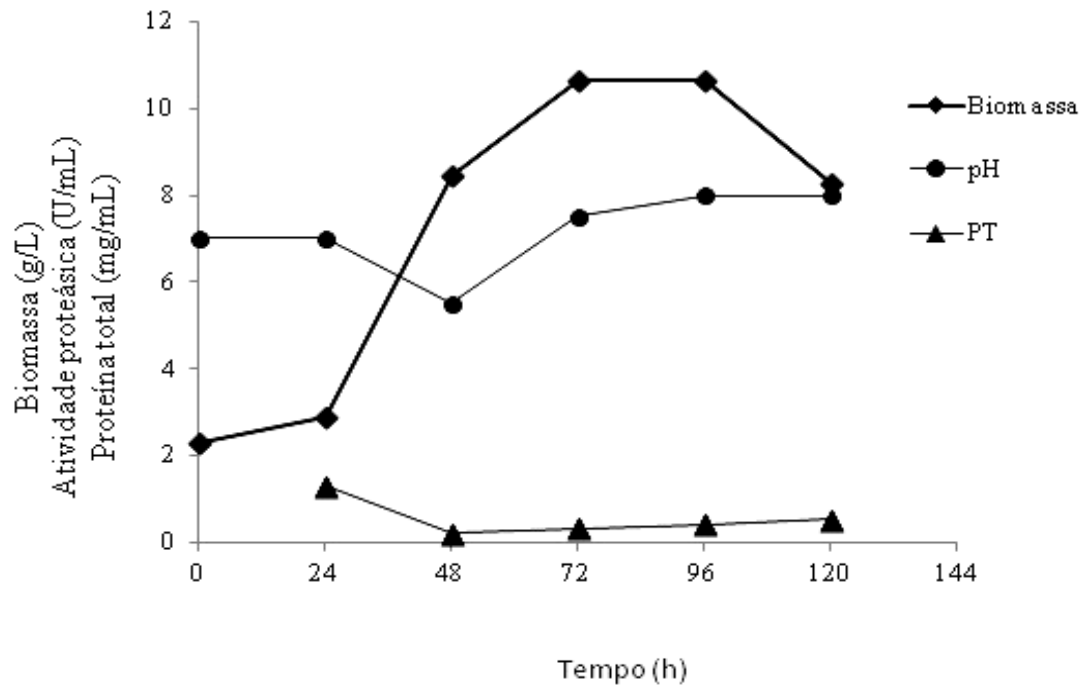

Figura 1- Curva de crescimento microbiano do Streptomyces sp. DPUA 1566 em meio MS-2. $\rightarrow-\mathrm{pH}^{-}$ $\rightarrow$ biomassa, $\rightarrow$ proteína total.

Apenas foi detectada atividade antimicrobiana no tempo 72 horas, ou seja, no final da fase exponencial e início da estacionária. Estes resultados estão de acordo com os descritos pela literatura. Yu et al. (1999) descreveram que a produção de metabólitos secundários pelos actinomicetos em meio líquido é limitada à fase estacionária, que frequentemente coincide com a escassez dos nutrientes do meio de cultura.

$\mathrm{O} \mathrm{pH}$ do meio, que inicialmente foi 7,0, elevouse a 8,0 no final da fermentação, o que corrobora os resultados apresentados por Carneiro da Cunha et al. (2010), que, ao utilizarem Nocardia sp. DPUA 1571, também em meio MS-2, tiveram o $\mathrm{pH}$ do meio de cultivo também aumentado de 7,0 para 8,5 ao final da fermentação.

O conteúdo proteico era elevado no início da fermentação, devido ao filtrado de farinha de soja, que é rico em proteínas, porém ocorreu uma diminuição das proteínas totais inicialmente, em razão da utilização dela pelo actinomiceto. Em seguida, foi observado um pequeno aumento no seu valor, possivelmente devido à produção de outros metabólitos microbianos de origem proteica.

Pode-se verificar que os ensaios fermentativos 3 e 4 (Tab. 3) não apresentaram atividade antimicrobiana e ambos possuem como característica comum a concentração de glicose 1\%. Esse comportamento foi confirmado por meio da análise estatística dos dados, a qual apresentou como única variável significativa a concentração de glicose, que apresentou um efeito negativo. Isso significa que o maior nível dessa variável foi prejudicial à produção de metabólitos antimicrobianos. 
Tabela 3. Resultados do planejamento fatorial $2^{2}$ para a produção de biocompostos com atividade antimicrobiana com 72 horas de fermentação

\begin{tabular}{cccccc} 
Ensaio & $\begin{array}{c}\text { Concentração de } \\
\text { filtrado de soja (\%) }\end{array}$ & $\begin{array}{c}\text { Concentração de } \\
\text { glicose }(\%)\end{array}$ & $\begin{array}{c}\text { Biomassa } \\
(\mathrm{g} / \mathrm{L})\end{array}$ & $\mathrm{pH}$ & $\begin{array}{c}\text { Atividade antimicrobiana } \\
\text { halo de inibição }(\mathrm{mm})\end{array}$ \\
\hline 1 & 1,0 & 0 & 1,5 & 8,0 & 29,7 \\
2 & 4,0 & 0 & 1,8 & 8,0 & 28,7 \\
3 & 1,0 & 1,0 & 10,4 & 5,0 & 0,0 \\
4 & 4,0 & 1,0 & 12,6 & 5,0 & 0,0 \\
$5(\mathrm{C})$ & 2,5 & 0,5 & 7,2 & 7,0 & 13,3 \\
$6(\mathrm{C})$ & 2,5 & 0,5 & 11,9 & 7,5 & 13,3 \\
$7(\mathrm{C})$ & 2,5 & 0,5 & 7,7 & 7,5 & 12,0 \\
$8(\mathrm{C})$ & 2,5 & 0,5 & 10,4 & 7,5 & 12,0 \\
\hline
\end{tabular}

(C) - Pontos centrais.

Sanchez e Demain (2002) afirmaram que, apesar de as fontes de carbono, como a glicose, serem comumente utilizadas para o melhor crescimento para produção de enzimas, antibióticos e outros metabólitos secundários, essa produção é frequentemente limitada devido à presença de um mecanismo regulador denominado de regulação do carbono catabólito (RCC). Este cataboliza rapidamente o suprimento de carbono e energia para o crescimento do microrganismo, reprimindo e esgotando o substrato primário, o que, consequentemente, prejudica a produção de enzimas e metabólitos secundários pelo microrganismo.

A Tab. 4 apresenta os efeitos estimados das variáveis e das interações entre elas (concentração de soja e concentração de glicose) sobre as respostas (atividade antimicrobiana, biomassa e $\mathrm{pH}$ ). Os valores com asterisco foram os efeitos estatisticamente significativos, com nível de confiança de 95\%. Fenômeno semelhante ao da atividade antimicrobiana ocorreu para o pH, ou seja, a concentração de glicose influenciou negativamente o $\mathrm{pH}$, apresentando efeito significativo e negativo. Quando a concentração de glicose foi $1 \%$, o pH do meio fermentado se acidificou ( $\mathrm{pH} 5,0)$, enquanto no meio sem adição de glicose o $\mathrm{pH}$ ficou alcalino (pH 8,0). Esse comportamento pode ser explicado pela utilização da glicose pelos Streptomyces para produção de ácidos pela via glicolítica, segundo Dekleva e Strohl (1987).

Tabela 4. Efeitos calculados com base nas respostas do planejamento fatorial $2^{2}$ para a produção de biocompostos com atividade antimicrobiana por Streptomyces sp. DPUA 1566

\begin{tabular}{cccc}
\hline Efeitos & Biomassa & $\mathrm{pH}$ & Halos de inibição \\
\hline $\begin{array}{c}\text { (1) Concentração de } \\
\text { filtrado de soja }\end{array}$ & 0,53 & 0,00 & $-0,64$ \\
$\begin{array}{c}\text { (2) Concentração de } \\
\text { glicose }\end{array}$ & $4,35^{*}$ & $-12,0^{*}$ & $-37,88^{*}$ \\
$(1) \quad \mathrm{X}(2)$ & 0,41 & 0,00 & 0,64 \\
\hline
\end{tabular}

*Efeito estatisticamente significativo (95\% de confiança).

Análise semelhante foi realizada para a resposta biomassa e verificou-se que também apenas a concentração de glicose apresentou efeito significativo, porém esse efeito foi positivo (Tab. 3 ), ou seja, a adição de glicose $1 \%$ ao meio de cultivo favoreceu o crescimento da biomassa. Tal resultado difere dos estudos realizados por outros autores, como Moreira et al. (2001), que, ao utilizarem o mesmo meio de cultura MS-2 no crescimento de Streptomyces clavuligerus, sem, entretanto, adicionar $1 \%$ de glicose ao meio de cultura, obtiveram um decaimento da biomassa após 50 horas de crescimento, o que ressalta a influência da glicose no aumento da biomassa.

\section{CONCLUSÕES}

Estes resultados permitem concluir que o Streptomyces sp. DPUA 1566 foi capaz de produzir biocompostos com atividade antimicrobiana capaz de inibir o crescimento de cepas patogênicas de Staphylococcus aureus isolados de caprinos portadores de mastite. A fonte de carbono (glicose) apresentou efeito 
negativo na fermentação do actinomiceto para a produção de metabólitos com atividade antimicrobiana, sendo sua ausência essencial para o desenvolvimento do biocomposto.

\section{AGRADECIMENTOS}

Os autores agradecem ao auxílio financeiro concedido pelo PNPD/Capes e pelo CNPq.

\section{REFERÊNCIAS}

AL-ZAHRAMI, S.H.M. Studies on antimicrobial activity of Streptomyces sp. Isolated from Jazan, JKAU: Sci., v.19, p.127-138, 2007.

BROWN, A.G.; BUTTERWORTH, D.; COLE, M. et al. Naturally occurring $\beta$-Lactam inhibitors with antibacterial activity. The J. of Antib., v.29, p.668-669, 1976.

CARNEIRO DA CUNHA, M.N.C.; SILVA, N.M.V.; TEIXEIRA, M.F.S. et al. Actinomicetos produtores de inibidores de $\beta$-lactamases com atividade antimicrobiana frente a isolados de mastite bovina; Arq. Bras. Med. Vet. Zootec., v.62, p.1312-1319, 2010.

CASTELLANI, A. Viability of some pathogenic fungi in distiled water. J. of Trop. Med. and Hygiene, v.42, p.225-226. In: MENEZES, M. e ASSIS, S.M.P. Guia prático para fungos fitopatogênicos, 2.ed., editora: Imprensa universitária da UFPE, 2004, p.79-80,1939.

CONTRERAS, A.; LUENGO, C.; SANCHEZ, A. et al. The role of intramammary pathogens in dairy goats. Livest. Prod. Sci., v.79, p.273-283, 2003.

CUNHA, A.P.; SILVA, L.B.G.; PINHEIRO JÚNIOR, J.W. et al. Perfil de sensibilidade antimicrobiana de agentes contagiosos e ambientais isolados de mastite clínica e subclínica de búfalas. Arq. Inst. Biol., v.73, p.17$21,2006$.

DEKLEVA, M.L.; STROHL, W.R. Glucose stimulated acidogenesis by Streptomyces peucetis. Can. J. Microbiol., v.33, p.1129-32, 1987.

ERICSSON, H.M.; SHERRIS, J.C. Antibiotic sensitivity testing-Report of an International Collaborative Study. Acta Pathol. Microbiol. Scand., v.217, p.1-90, 1971.
FOSTER, T.J. Immune evasion by Staphylococci. Nat. Rev. Microbiol., v.3, p.948-958, 2005.

FRANÇA, C.A.; PEIXOTO, R.M.; CAVALCANTE, M.B. et al. Antimicrobial resistance of Staphylococcus spp. From small ruminant mastitis in Brazil. Pesq. Vet. Bras., v.32, p.747-753, 2012.

FREITAS, M.F.L.; PINHEIRO JUNIOR, J.W.; STAMFORD, T.L.M. et al. Perfil de sensibilidade antimicrobiana in vitro de Staphylococcus coagulase positivos isolado de leite de vacas com mastite no agreste do estado de Pernambuco. Arq. Inst. Biol., v.72, p.171-177, 2005.

GAMA, E.V.G.; GARRIDO, M.S.; SILVA, F. et al. Produção de biomassa de erva-cidreira [Lippia alba (Mill.) N.E.Br.] sob adubação com composto de capim elefante inoculado e sem inoculação de actinomicetos. Rev. Bras. Pl. Med., v.14, p.163-168, 2012.

MITRA, A.; PRAMANIK, A.; SANTRA, S.C. et al. Phylogeny, phenotypic and nutritional characteristics of estuarine soil actinomycetes having broad-spectrum antimicrobial activity derived from an ecologically guided bioprospecting programme. World J. Microbiol. Biotechnol.,v.27, p.1679-1688, 2011.

MOREIRA, K.A.; CAVALCANTI, M.T.H; DUARTE, H.S. et al. Partial characterization of proteases from Streptomyces clavuligerus using an inexpensive medium, Braz. J. Microbiol., v.32, p.215-220, 2001.

MORK, T.; KVITLE, B.; MATHISEN, T. et al. Bacteriological and molecular investigations of Staphylococcus aureus in dairy goats. Vet. Microbiol., v.141, p.134-141, 2010.

MOTA, R.A; MEDEIROS, E.S.; SANTOS, M.V. et al. Participação dos Staphylococcus spp na etiologia das mastites em bovinos leiteiros no estado de Pernambuco (Brasil). Cienc. Anim. Bras., v.13, p.124-130, 2012.

NATIONAL Comité Clinical Laboratory Standards - NCCLS document M7-A6 [ISBN 156238-486-4]. NCCLS, 940 West Valley Road, Suite 1400, Wayne, Pennsylvania 19087-1898 USA. 2003. 
NEVES, P.B.; MEDEIROS, E.S.; SÁ, V.V. et al. Perfil microbiológico, celular e fatores de risco associados à mastite subclínica em cabras no semiárido da Paraíba. Pesq. Vet. Bras., v.30, p.379-384, 2010.

PACHECO DA ROSA, J.; KORENBLUM, E. et al. Streptomyces lunalinharesii strain 235 shows the potential to inhibit bacteria involved in biocorrosion processes. BioMed Res. Internat., art. no. 309769, 10p., 2013.

PARK, Y.H.; LEE, S.U.; FERENS, W.A. et al. Unique features of bovine lymphocytes exposed to a Staphylococcal enterotoxin. J. Vet. Sci., v.7, p.233-239, 2006.

PEIXOTO, R.M.; PEIXOTO, R.M.; LIDANI, K.C.F. et al. Genotipificação de isolados de Staphylococcus epidermidis provenientes de casos de mastite caprina . Cienc. Rural, v.43, p. 322-325, 2013.

PFEFFERLE, C.; THEOBALD, U.; GÜRTLER, $\mathrm{H}$. et al. Improved secondary metabolite production in the genus Streptosporangium by optimization of the fermentation conditions. $J$. Biotechnol., v.80, p.135-142, 2000.

PORTO, A.L.F.; CAMPOS-TAKAKI, G.M.; LIMA FILHO, J.L. Effects of culture conditions on protease production by Streptomyces clavuligerus growing soy bean flour médium. Appl. Biochem. Biotechnol., v.60, p.115-122, 1996.

PRIDHAM, T.G.; ADERSON, P.; FOLEY, C. et al. Selection of Media for Maintenance and Taxonomic Study of Streptomyces. Antibiot. M., p.947-953, 1957.

RANG, H.P.; DALE, M.M.; RITTER, J.M. Farmacologia. 5.ed. Rio de Janeiro: Editora Guanabara Koogan, 2003. p.470-478.
RIBEIRO, M.E.R.; PETRINI, L.A.; AITA, M.F. et al. Relação entre mastite clinica, subclinica infecciosa e não infecciosa em unidades de produção leiteiras na Região Sul do Rio Grande do Sul. Rev. Brasil. Agrociência, v.9, p.287-290, 2003.

SAHIN, N.; UGUR, A. Investigation of the antimicrobial activity of some Streptomyces isolates. Turk J. Biolog., v.27, p.79-82, 2003.

SANCHEZ, S.; DEMAIN, A.L. Metabolic regulation of fermentation processes. Enz. Microbiol. Tech., v.31, p.895-906, 2002.

SILVEIRA, G.P.; NOME, F.; GESSER, J.C. et al. Estratégias utilizadas no combate à resistência bacteriana. Quim. Nova, v.29, p.844-855, 2006.

STATSOFT INC. STATISTICA (data analysis software systems) version 8.0. 2008.

TAPONEN S.; PYORALA, S. Coagulasenegative staphylococci as cause of bovine mastitis-Not so different from Staphylococcus aureus? Vet. Microbiol., v.134, p.29-36, 2009.

TOZZETI, D.S.; BATAIER, M.B.N.; ALMEIDA, L.R. Prevenção, controle e tratamento das mastites bovinas - Revisão de literatura. Rev. Cienc. Elet. Med. Vet., v.10, p.1679-7353, 2008.

VIJAYAKUMAR, R.; PANNEERSELVAM， K.; MUTHUKUMAR, C. et al. Optimization of Antimicrobial Production by a Marine Actinomycete Streptomyces afghaniensis VPTS3-1 Isolated from Palk Strait, East Coast of India. Indian J. Microbiol., v.52, p.230-239, 2012.

YU, T.W.; SHEN, Y.; DOI-KATAYAMA, Y. Direct evidence that the rifamycin polyketide synthase assembles polyketides chains processively. Proc. Natl. Acad. Sci. USA, v.96, p.9051-9056, 1999. 